\title{
The Research on the Enhanced Effect of Dual-frequency
}

\author{
Ping Zhang ${ }^{1}$, Ronghui Lin $^{1}$, Zhe Huang ${ }^{1}$, Xiangjun Wang ${ }^{1}$, Hui You ${ }^{2 *}$, Jun \\ $\mathrm{Gao}^{2}$, Xiaofei Wang ${ }^{2}$ \\ ${ }^{1}$ Department of Automation, University of Science and Technology of China, Hefei 230026, China \\ ${ }^{2}$ Institute of Intelligent Machines, Chinese Academy of Science, Hefei230031, China
}

Keywords: Dual frequency; Therephthalate dosimetry; Acoustic cavitation; Enhanced effect

\begin{abstract}
An ultrasonic reactor has been built to understand the augment of ultrasonic cavitation effect under dual frequency. Therephthalate dosimetry has been performed to determine the sonolytic yields of the $\mathrm{OH}$ free radicals which are produced in aqueous solution after ultrasonic irradiation. In this paper, two sets of dual-frequency ultrasound irradiation experiments composing of $20 \mathrm{kHz}$ and $25 \mathrm{kHz}, 20 \mathrm{kHz}$ and $1.106 \mathrm{MHz}$, respectively, have been carried out. The studies indicated that dual-frequency ultrasonic irradiation can significantly enhance the cavitation effect, the acoustic cavitation yield were significantly greater compared to single frequency irradiation.
\end{abstract}

\section{Introduction}

Sonochemistry first started in the mid-1980s as a new of intersectional research to accelerate the chemical reaction or to change reaction course with ultrasound (Ruo et al. 1992). When ultrasound propagates in liquid media, the molecule distance of the media can exceed a critical value above led to the interaction between molecules are interrupted, and micro bubble will be generated (Leighton et al 1994, Young et al. 1989). These micro cavitation bubbles expand and shrink periodically. During the compression phase of the bubble motion, volume of the bubbles is reduced. Some bubbles disappear at this stage. Since those bubbles cannot exist permanently if they were not at resonant state. The pressure within the bubble is inadequate to withstand the pressure in the liquid media, thus these bubbles collapse, this kind of process is called a cavitation phenomenon. Some researches indicated that the sonochemical effects are attributed to this phenomenon. Ultrasound is widely used in biomedical, synthetic chemistry, atomization, emulsification, cleaning, degassing, solder, extraction, drilling, homogenization etc. (Mason et al. 1988, Suslick et al. 1988). In aqueous systems, water molecule is cleaved into $\mathrm{H}$ and $\mathrm{OH}$ radicals, with other species exist, various other radicals may form (Lida et al. 2005, Fang et al. 1996). For both fundamental research and industrial applications, there is a desire to improve the efficiency and efficacy of the system used.

In an instant and extreme condition, there are many factors that can affect the cavitation such as vapor pressure, temperature, surface tension, viscosity, and dissolved gas (Hung et al. 2005). Asakura et al. invested effect of ultrasonic frequency and liquid height on sonochemical efficiency (Asakura et al. 2008) by using four different ultrasonic frequencies. They found that the sonochemical efficiency depended on the frequency and liquid height. Huang et al. reported the yield of cavitation in liquid can reach a maximum value at an optimum ultrasonic frequency (Huang et al. 1995). In this paper, two sets of dual-frequency ultrasound irradiation composing of $20 \mathrm{kHz}$ and $25 \mathrm{kHz}, 20 \mathrm{kHz}$ and $1.106 \mathrm{MHz}$, respectively, are firstly investigated. Then ultrasonic cavitation yield was investigated by using the above two ultrasonic frequency alone. By comparing the above two results, the enhancement of ultrasonic cavitation effect was observed. Terephalate dosimetry has characteristics such as high sensitivity, easy acquisition, easy measurement, etc. Terephthalate dosimetry has been carried out to determine the ultrasonic cavitation yield.

\section{Experimental}

\section{Sonication apparatus}

A schematic experiment setup is shown in Fig.1. As shown in the picture, the two setups are made up of two separate ultrasonic devices. As you can see in the picture, in this paper, the 
experiment used a total of three kinds of transducer: a $20 \mathrm{kHz}$ ultrasonic probe, a $25 \mathrm{kHz}$ ultrasonic cleaner and a $1.106 \mathrm{MHz}$ high frequency focused ultrasound transducer. Setup A was a dualfrequency ultrasound system made up of $20 \mathrm{kHz}$ ultrasonic probe and $25 \mathrm{kHz}$ ultrasonic cleaner. Setup B was a dual-frequency ultrasound system made up of $20 \mathrm{kHz}$ ultrasonic probe and 1.106 $\mathrm{MHz}$ high frequency focused ultrasound transducer. Ultrasonic probe has two selectable intensities: $90 \%$ duty ratio and $50 \%$ duty ratio. The input circuit parameters of the ultrasonic amplitude transformer is AC220V, $50 \mathrm{kHz}$, and the output circuit parameters is $20 \mathrm{kHz}, 100 \mathrm{~W}$. The power of the ultrasonic cleaning machine is adjustable with the maximum power being $5 \mathrm{~W}$. In the second setup, the containers of the sample solution was made of a cylindrical acrylic pipe with thickness and inner diameter of 2 and 25mm,respectively; The length of the pipe is $125 \mathrm{~mm}$
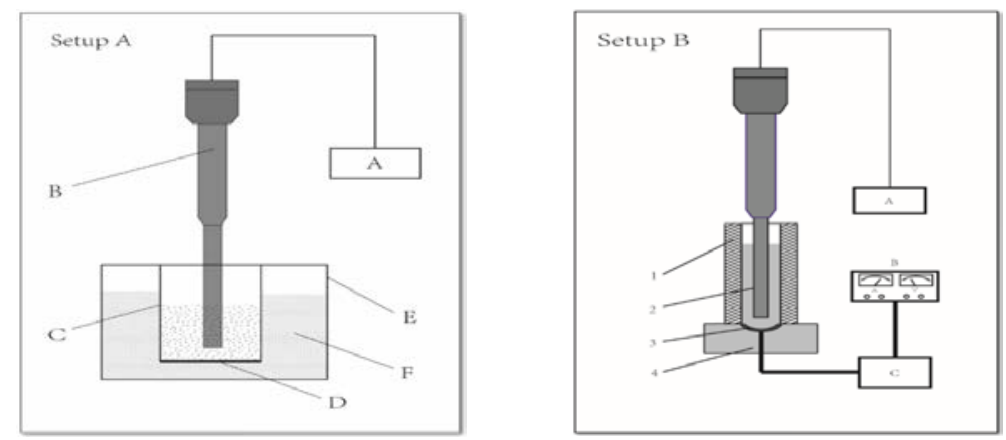

Fig.1. Schematic diagrams of experiment set-up. Setup A: A: ultrasonic generator; B: ultrasonic probe type; C: organic class tube; D: plant the sealing membrane; E: ultrasonic cleaners; F: water. Setup B: 1: Organic class tube; 2 sample solution; 3: Transducer; 4: polydimethylsiloxane; A: ultrasonic generator; B: single generator; C: power amplifier

\section{Chemical solution}

In this paper, therephalate dosimetry was selected as testing material to characterize ultrasonic yield. The terephthalic acid(TA) was prepared with the following composition: TA $0.166 \mathrm{~g}, \mathrm{NaOH}$ $0.100 \mathrm{~g}$, and phosphate buffer ( $\mathrm{PH} 7.4$ ), prepared from $\mathrm{kH}_{2} \mathrm{PO}_{4} 0.2995 \mathrm{~g}$ and $\mathrm{NaHPO}_{4}$ The resulting solution gave a concentration with respect to TA of $2 \times 10^{-3} \mathrm{~mol} / \mathrm{L}$ Detection of 2hydroxyterephalate was by fluorimetry (xinghao) with an excitation wavelength of $315 \mathrm{~nm}$ and an emission wavelength of $425 \mathrm{~nm}$. When using this method, the samples must be sufficiently diluted to avoid interference by the optical absorption of the reagents.

\section{Quntifaction of chemical reaction}

Water can produce hydrogen and hydrogen under ultrasonic radiation that lead to an oxidation reaction with the gases or solutions dissolved in aqueous solution. From the earlier studies, it was found that terephthalic method (Mason et al. 1994) has advantages such as simple operation and high sensitivity. Therefore, terephthalic dosimetry was used to evaluate ultrasonic cavitation yield. Measurements were repeated several times and were found to be quite reproducible at a fixed location. The data reported is averaged over at least 4 experimental runs.

\section{Result and discussion}

\section{Cavitation effect of single frequency of ultrasonic and dual-frequency composite ultrasonic under the condition of the same power input}

Fig.2.a and Fig.2.b was mapped under the condition of ultrasonic horns, the transducer's ratio of on/off was 9:1(90\% duty ratio) and 1:1(50\% duty ratio),respectively. As shown in fig.2, it can be seen that dual-beam ultrasonic irradiation can produce more cavitation yield than the algebraic sum of the amount produced by two individual sonications. When ultrasonic horns transducer's ratio of on/off of the pulse was 9:1, for setup A, the amount produced by dual-ultrasonic beam was about 2.53 times than the algebraic sum of the two individual sonication; For setup B, the amount produced by $20 \mathrm{Khz}$ combined with $1.106 \mathrm{Mhz}$ ultrasonic irradiation was about 1.32 times than 
algebraic sum of the production of the two different transducers were applied successively. When ultrasonic horns transducer's ratio of on/off of the pulse was 1:1, for setup A and setup B, the mount produced by dual-ultrasonic beam were about 3.13 and 1.37 than algebraic sum of the two individual sonications.
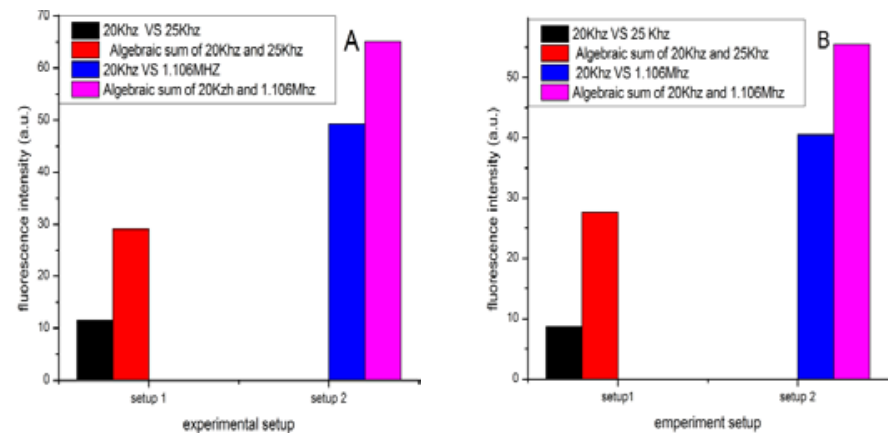

Fig.2. Effects of ultrasonic operation modes on cavitation.

Fig.2.a: Ultrasonic horns circuit work under the condition of 90\% duty cycle; Fig2.b: Ultrasonic horns circuit work under the condition of $50 \%$ duty cycle.

We can see from the experimental results that cavitation yield can be augmented during two combined ultrasound frequency. When two ultrasound frequencies are applied successively, they affect the liquid independently. But when two ultrasound frequencies are combined, the solution reacts more violently, dissolving more air, which leads to more cavitation nuclei, in this way, cavitation yields boosts. The results obtained from the two experiment setups conform to earlier report (Zhu et al. 1998).

\section{The impact of transducer's ratio of on/off of the pulse on the cavitation yield}

From the previous experiments we can see, the cavitation yield is higher when two frequency ultrasounds are applied jointly than successively. As shown in fig.2.a, when two different frequencies are applied successively, cavitation yield in $90 \%$ duty cycle is 1.32 times higher than that of $50 \%$. Whereas for setup B, cavitation yield is 1.21 times higher in $90 \%$ duty cycle than $50 \%$. For setup A, when two different frequencies applied jointly, cavitation yield with $90 \%$ duty cycle is 1.32 times higher than that $50 \%$. However for setup B, cavitation yield is 1.17 times higher when the duty cycle is $90 \%$ than $50 \%$.

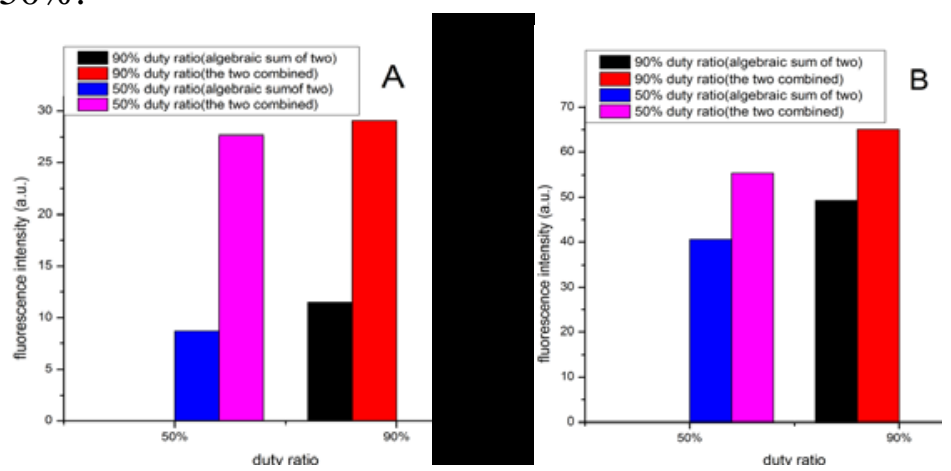

Fig.3. Fig.3.a:20KZH and 25KHZ dual-frequency ultrasonic cavitation yield;

Fig.2.b 20KZH and 1.106MHZ dual-frequency ultrasonic cavitation yield.

The experiment result in Fig.3 demonstrates that the cavitation yield of the sample solution is significantly higher when ultrasonic horns transducer's ratio of on/off was 9:1(90\% duty ratio) than that of $1: 1(50 \%$ duty ratio), whether in dual-frequency ultrasound irradiation or two individual irradiations. We can explain the result by the follow equation:

$$
\mathrm{U}=\mathrm{V}_{\mathrm{pp}} /(2 *(1-\alpha))
$$

Where $\mathrm{U}, \mathrm{V}_{\mathrm{pp}}$ and $\alpha$ denote the effective square-wave voltage value, voltage peak and duty ratio, respectively. Square wave effective voltage value of $50 \%$ ratio is a quarter of the effective squarewave voltage value, while square wave voltage effective value of $90 \%$ duty ratio if a half of the 
effective square-wave voltage value. When the load is fixed, the output energy value of square wave can be represented as follow equation:

$$
\mathrm{E}=\mathrm{u}^{2} /(\mathrm{R} * \mathrm{t})
$$

Where $\mathrm{E}$ is output energy of the square wave and $\mathrm{R}$ constant load, $\mathrm{u}$ is the corresponding effective voltage value under various duty ratios. The output energy of square wave can be obtained by the above equation. The output energy of square wave at $50 \%$ duty ratio is $0.0625 \mathrm{U} 2 \mathrm{t} / \mathrm{R}$, while that of $90 \%$ duty ratio is $0.2025 \mathrm{U}^{2} \mathrm{t} / \mathrm{R}$. Under the same work time, the total energy transducer can emit under the condition of $90 \%$ duty ratio is 3.24 times than the energy value can be received under the condition of $50 \%$ duty ratio. At the same action time of ultrasonic irradiation, the more energy transducer the transducer receives, the stronger the cavitation effective might be.

\section{Ultrasonic irradiation time on the influence of cavitation yield}

The experiments to study the cavitation yield under single ultrasonic frequency reported by CHEN Zhaohua (CHEN et al. 1997) and his colleagues showed that cavitation effect (that is, the sonochemical yield) grow linearly with the increase of ultrasonic irradiation time. Cavitation yield's linear increase can be explained by the accumulation of the cavitation effect. Figure 5 shows that ultrasound radiation between the $2 \mathrm{~min}$ to $10 \mathrm{~min}$ for experimental setup, the fluorescence intensity of the sample solution increased with the extension of time. From the fig.5, we can see that the time course deviated slightly from linearity at longer irradiation time. There are many factors affecting the ultrasonic cavitation such as coefficient of viscosity, coefficient of surface tension, vapor pressure, solution temperature, number of species dissolved in the liquid and so on (WANG et al. 2003). As Slimane Merouani et al. (Merouani et al. 2010) said the temperature of sample solution rise would cause cavitation effect to be weakened. When the ultrasonic irradiation time increases, a lot of physical factors changed in the sample solution, thus resulting in cavitation intensity weaken.
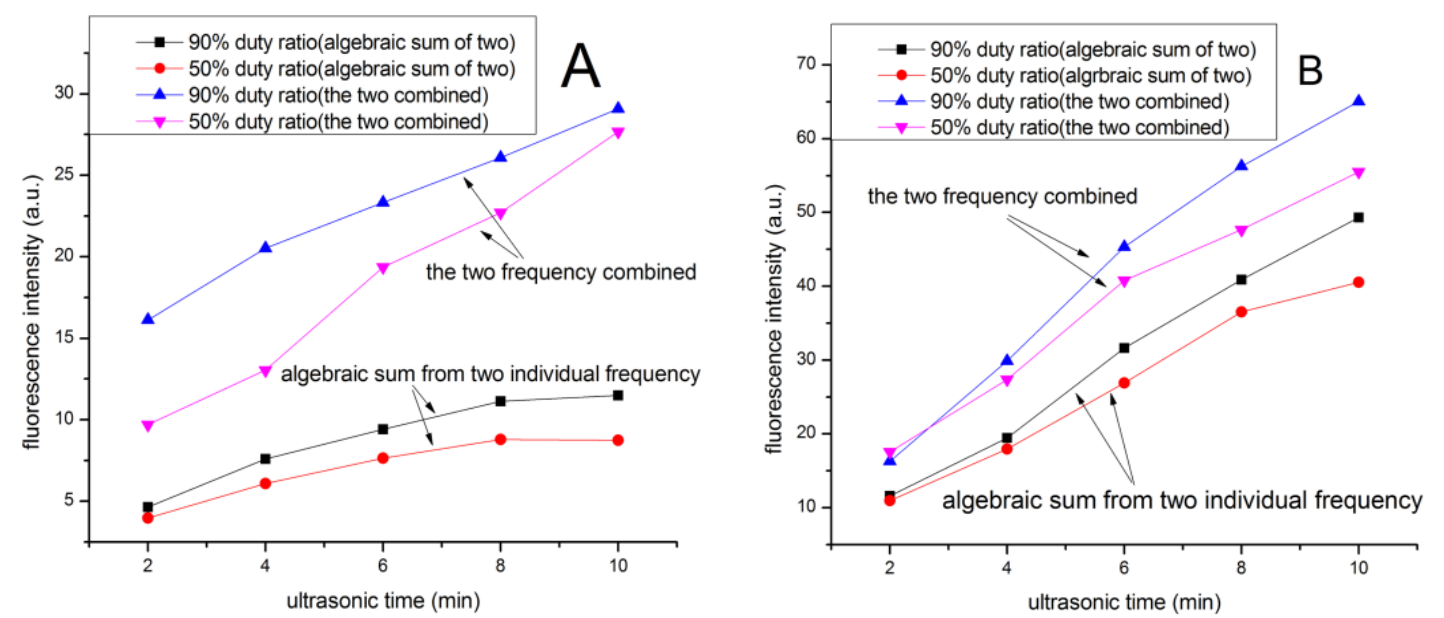

Figure.4. Fluorescence intensity changes of TA solution with ultrasonic irradiation time changes.

Figure.5.a. In 20 kHz sonication combined with 25 kHz ultrasonic; Fig.5.b. In 20kHz sonication combined with $1.106 \mathrm{MHz}$ ultrasonic.

\section{Conclusion}

Terephthalic dosimetry provides a sensitive method for the study of sonochemical effects. Several conclusions can be attained through the above experiments. Firstly, dual-frequency ultrasonic irradiation has enhancement effect over ultrasound cavitation. In other words, the combing irradiation of dual-frequency ultrasound can significantly increase the cavitation yield compared with two individual ultrasonic irradiations. Dual-frequency irradiation system can make the liquid sample undergo stronger mechanical disturbance and dissolve more air through the liquid surface into the sample liquid, thus resulting in more cavitation nuclei in solution. Secondly, the actual energy acts on the transducer is different under the conditions of different duty ratio. Thus, under the same action time of ultrasonic irradiation, the more energy the transducer emits, the stronger the cavitation effect can be. Thirdly, with the increase of ultrasonic action time, cavitation 
yield has a linear growth. Cavitation yield linear increase can be explained by the results of accumulation of the cavitation. When the ultrasonic irradiation time increases, a lot of physical factors change in the sample solution, thus resulting in cavitation intensity to weaken. Thus, we can see that the time course deviated slightly from the linearity at longer irradiation time.

\section{Acknowledgements}

This work is supported by National Natural Science Foundation of China (No. 61176105) and Hundred Talents Program of Chinese Academy of Science.

\section{References}

[1] Asakura .Y, Nishida .T, Matsuoka. T, Koda. S 2008. Effects of ultrasonic frequency and liquid height on sonochemical efficiency of large-scale sonochemical reactors. Ultrason. Sonochemistry15: 244-250.

[2] CHEN Zhaohua, ZHU Changping, ZHAO Yiyun, et al.1997. Study on the sonochemical yield of low frequency ultrasound by iodine release method. Technol.Acoust.16:191-193.

[3] F.R. Young 1989. Cavitation. London: McGraw-Hill.

[4] Huang J, Feng R, Zhu C, et al. 1995. Low-MHZ frequency effect on a sonochemical reaction determined by an electrical method. Ultrason.Sonochemistry:S93-S97.

[5] K.S. Suslick 1988. Ultrasound- Its Chemical, Physical and Biological Effects. New York : VCE, Publishers.

[6] L.B Hung, S.J. Wu, F.M. Zhou, Several factors affecting sonochemical yield. Technol. Acoust.24(2005)210-214.

[7] Slimane Merouani, Oualid Hamdaoui, Fethi Saoudi, Mahdi Chiha 2010. Influence of experimental parameters on sonochemistry dosimetries: KI oxidation, Fricke reaction and H2O2 production, Journal of Hazardous Materials 178:1007-1014.

[8] T.J. Mason and J.P. Lorimer 1988. Theory Applications and Uses of Ultrasound in Chemistry. Chichester: Ellis Horwood.

[9] T.G. Leighton 1994. The Acoustic Bubble. London: Academic Press.

[10] T.J Mason, J.P Lorimer, D.M. Bates and Y. Zhao, et al.1994. Dosimetry in sonochemistry: The use of aqueous terephthalate ion as a fluorescence monitor. Ultrason.Sonochem.1: 91-95

[11] WANG Ping-hui 2003. A study and analysis on influencing factor of the ultrasonic cavitation. Journal of Hebei Institute of Technology. 25:154-161

[12] X.Fang, G.Mark, C.von Sonntag 1996. OH radical formation by ultrasound in aqueous solutions.1.The chemistry underlying the terephthalate dosimeter. Ultrason. Sonochem.3:57-63

[13] Y.lida, K. Yasui,T. Tuziuti, M. Sivakummar 2005. Sonochemistry and its dosimetry. Microchemical journal.80:159-164

[14] Zhu Changping FengRuo, Chen Zhaohua, et al. 1998. The sonochemical yield of bifrequency irradiation and its frequency effect. Journal of Nanjing University 34:94-96 